\title{
Impact of pre-pregnancy diabetes mellitus on congenital anomalies, Canada, 2002-2012
}

\author{
S. Liu, MB, PhD (1); J. Rouleau (1); J. A. León, MD (1); R. Sauve, MD (2); K. S. Joseph, MD, PhD (3, 4, 5); \\ J. G. Ray, MD (6); Canadian Perinatal Surveillance System (1)
}

This article has been peer reviewed.

Tweet this article

Abstract

Objective: To examine the impact of pre-pregnancy diabetes mellitus (DM) on the population birth prevalence of congenital anomalies in Canada.

Methods: We carried out a population-based study of all women who delivered in Canadian hospitals (except those in the province of Quebec) between April 2002 and March 2013 and their live-born infants with a birth weight of 500 grams or more and/or a gestational age of 22 weeks or more. Pre-pregnancy type 1 or type 2 DM was identified using ICD-10 diagnostic codes. The association between DM and all congenital anomalies as well as specific congenital anomaly categories was estimated using adjusted odds ratios; the impact was calculated as a population attributable risk percent (PAR\%).

Results: There were 118892 infants with a congenital anomaly among 2839680 live births (41.9 per 1000). While the prevalence of any congenital anomaly declined from 50.7 per 1000 live births in 2002/03 to 41.5 per 1000 in 2012/13, the corresponding PAR \% for a congenital anomaly related to pre-pregnancy DM rose from $0.6 \%$ ( $95 \%$ confidence interval [CI]: $0.4-0.8$ ) to $1.2 \%$ (95\% CI: 0.9-1.4). Specifically, the PAR \% for congenital cardiovascular defects increased from $2.3 \%(95 \% \mathrm{CI}: 1.7-2.9)$ to $4.2 \%(95 \%$ CI: 3.5-4.9) and for gastrointestinal defects from $0.8 \%(95 \% \mathrm{CI}: 0.2-1.9)$ to $1.4 \%$ (95\% CI: $0.7-2.6)$ over the study period.

Conclusion: Although there has been a relative decline in the prevalence of congenital anomalies in Canada, the proportion of congenital anomalies due to maternal prepregnancy DM has increased. Enhancement of preconception care initiatives for women with DM is recommended.

Keywords: diabetes mellitus, pre-pregnancy, congenital anomaly, birth defect, trends, population attributable risk percent

\section{Introduction}

Major congenital anomalies affect approximately $3 \%$ to $5 \%$ of newborn infants in Canada and are the leading contributor to perinatal and infant mortality. ${ }^{1-3}$ Congenital anomalies may also necessitate surgery and special care for several years after birth. ${ }^{4}$
Children of women with pre-pregnancy type 1 and type 2 diabetes mellitus (DM) are at 2 to 10 times higher risk for congenital anomalies, especially cardiovascular and central nervous defects, compared with the nondiabetic population. ${ }^{5-11}$ Given that the rate of pre-pregnancy DM has doubled over the past decade or so, ${ }^{12}$ clinical and public health programs in
Key findings

- Among Canadian women aged 35 year or more, pre-pregnancy diabetes mellitus almost doubled, from 6.4 per 1000 live births in 2002 to 12.3 per 1000 live births in 2012 .

- The proportion of congenital anomalies attributable to maternal pre-pregnancy diabetes mellitus has increased over the last decade.

- $4.2 \%$ of congenital heart defects in infants were attributable to prepregnancy diabetes mellitus in 2012, compared with $2.3 \%$ in 2002 .

- Increased proportions of congenital gastrointestinal, genitourinary and musculoskeletal defects were also attributed to pre-pregnancy diabetes mellitus.

particular would benefit from knowing if the effect of pre-pregnancy DM on the rate of congenital anomalies has also changed.

Preconception care is recommended for women with pre-pregnancy DM, including periconceptional glycemic control, optimal nutrition intake and folic acid supplementation. ${ }^{13}$ In addition, early prenatal diagnosis of lethal congenital anomalies and subsequent pregnancy termination play an important role in the management of pregnancy among women with pre-pregnancy DM. ${ }^{14}$

In this study we examined the impact of pre-pregnancy DM on the population birth prevalence of congenital anomalies in

Author references:

1. Maternal, Child \& Youth Health Unit, Surveillance and Epidemiology Division, Centre for Chronic Disease Prevention, Public Health Agency of Canada, Ottawa, Ontario, Canada 2. Departments of Pediatrics and Community Health Sciences, University of Calgary, Calgary, Alberta, Canada

3. Department of Obstetrics \& Gynaecology, University of British Columbia, Vancouver, British Columbia, Canada

4. Children's and Women's Hospital of British Columbia, Vancouver, British Columbia, Canada

5. School of Population and Public Health, University of British Columbia, Vancouver, British Columbia, Canada

6. Departments of Medicine, Health Policy Management and Evaluation, and Obstetrics and Gynecology, St Michael's Hospital, University of Toronto, Toronto, Ontario, Canada

Correspondence: Shiliang Liu, Room 405A2, AL 6804A, 785 Carling Ave., Ottawa, ON K1A 0K9; Email: shiliang.liu@phac-aspc.gc.ca 
Canada. Specifically, we used the population attributable risk percent (PAR\%). This index quantifies the proportion of congenital anomalies attributable to prepregnancy DM that can be potentially prevented by optimizing DM control.

\section{Methods}

We completed a population-based cohort study of all live births in Canada (excluding the province of Quebec) for the fiscal years 2002/03 through 2012/13. We obtained information on each hospital birth from the Discharge Abstract Database (DAD) of the Canadian Institute for Health Information (CIHI). The DAD contains the abstracted and collated information on each hospitalization in Canada excluding Quebec. This information is extracted by trained medical archivists in each hospital and coded according to a standard protocol. Hospitalization records for the mother's delivery and her live-born infant's birth were deterministically linked using a common mother-newborn number. The DAD data file contains information on demographics, health service use, diagnosis and procedure. Information in the DAD has been previously validated ${ }^{15}$ and extensively used in research ${ }^{6,16,17}$ and in a number of Canadian perinatal surveillance reports, ${ }^{18}$ including congenital anomalies surveillance reports. ${ }^{1}$

We included all mother-newborn pairs, except for births at less than 22 weeks' gestation or less than 500 grams birthweight. Variables used in the study included maternal province of residence, three-digit postal code, residence code, year of delivery, parity, newborn's sex, delivery admission and discharge date, newborn date of death (if applicable), birth weight, and whether the delivery resulted in a live birth or a stillbirth. However, stillbirths were not included in the study population because stillbirth records could not be linked to the mother's records. Pre-pregnancy DM (either type 1 or type $2 \mathrm{DM}$ ) and congenital anomalies were identified based on information in the 25 diagnostic fields of the medical record, coded according to the International Statistical Classification of Diseases and Related Health Problems, 10th Revision, Canada (ICD-10 CA). The diagnosis of a congenital anomaly was based on hospital records at the birth hospitalization and also hospital readmission within 30 days of birth. ${ }^{1,18}$ We used a melding process to categorize congenital anomalies in the DAD to group the admission of the same infant into one record and avoid duplication. ${ }^{1}$ This process included a summary by individual ICD-10 codes, 59 standard categories and 14 major categories (i.e. a few congenital anomalies categories combined). A congenital anomalies subcategory was defined by a list of one or more ICD-10 diagnostic codes, and included every infant with one or more of the codes listed within a given category. For example, an infant with an upper limb deficiency and a lower limb deficiency was counted as one case of limb deficiency. However, the upper and lower limb anomalies could also be reported as 2 separate categories because they had 2 distinct ICD-10 CA codes. Similarly, infants with more than one anomaly involving different categories were counted in each of those categories as a single case.

We calculated the prevalence of all congenital anomalies and the prevalence of each subcategory of interest. We then determined the prevalence of congenital anomalies among women with pre-pregnancy DM. Crude and adjusted odds ratios (OR) and $95 \%$ confidence intervals (CI) were estimated for the association between prepregnancy DM and congenital anomalies. We used multivariate logistic regression to adjust for maternal age $(<20,20-24$, $25-29,30-34,35-39$ and $\geq 40$ years), parity (i.e. number of previous births: 0, 1, 2, 3+, missing), and the year of delivery. We also estimated the proportion of cases of congenital anomaly that would be eliminated if a risk factor such as pre-pregnancy DM (assumed to be causally associated with congenital anomalies) was removed from the population using the adjusted effect measure in the following equation:

$$
\text { pd }(\mathrm{OR}-1) / \mathrm{OR} \text {, }
$$

where $p d$ is the proportion of cases exposed to the risk factor, and $O R$ is the adjusted OR attributable to the risk factor. ${ }^{19}$ The adjusted OR expressing the relation between pre-pregnancy DM and congenital anomalies was estimated separately for each year. Since prevalence changes, pre-pregnancy DM may have led to changes in the strength of the relation between pre-pregnancy DM and congenital anomalies. All statistical analyses were performed with SAS Unix version 9.2 (SAS Institute, Cary, NC).

This study was carried out by the Public Health Agency of Canada, which has a federal mandate to monitor the health of the Canadian population. The data analysed were de-identified and ethics review board approval was not required.

\section{Results}

The study population included 2839680 live births. The prevalence of pre-pregnancy DM among mothers of live-born infants increased significantly over the 11 years of the study period, from 4.6 per 1000 live births in $2002 / 03$ to 7.5 per 1000 live births in 2012/13. The prevalence of type $1 \mathrm{DM}$ among the mothers of live-born infants remained unchanged, from 2.7 per 1000 live births in $2002 / 03$ to 2.8 per 1000 live births in $2012 / 13$ ( $p$ value for linear trend $=.43$ ), while the prevalence of maternal type $2 \mathrm{DM}$ increased from 1.9 per 1000 live births to 4.7 per 1000 live births in 2012/13 ( $p$ value for linear trend $<.0001$ ). Overall, among live births to women aged 35 years or more, pre-pregnancy DM rates almost doubled, from 6.4 per 1000 to 12.3 per 1000 live births from 2002/03 to 2012/13 (Figure 1).

A total of 118892 infants with one or more congenital anomalies were identified among the 2839680 infants born during the study period-equivalent to 41.9 congenital anomalies per 1000 live births. The prevalence of any congenital anomalies declined from 50.7 per 1000 live births in $2002 / 03$ to 38.5 per 1000 in $2007 / 08$, then rose to 41.5 per 1000 in $2012 / 13$. The prevalence of most of the major congenital anomaly categories, for instance, musculoskeletal defects and cardiovascular defects, declined over this entire period while genitourinary defects increased (Figure 2).

The rate of any congenital anomaly was 93.4 per 1000 live births among women with pre-pregnancy DM versus 41.5 per 1000 live births among women without prepregnancy DM. Rates were similar among women with type 1 DM (93.7 per 1000 live 
FIGURE 1

Trends in pre-pregnancy diabetes mellitus according to the age of the mother, Canada (excluding Quebec), 2002/03-2012/13

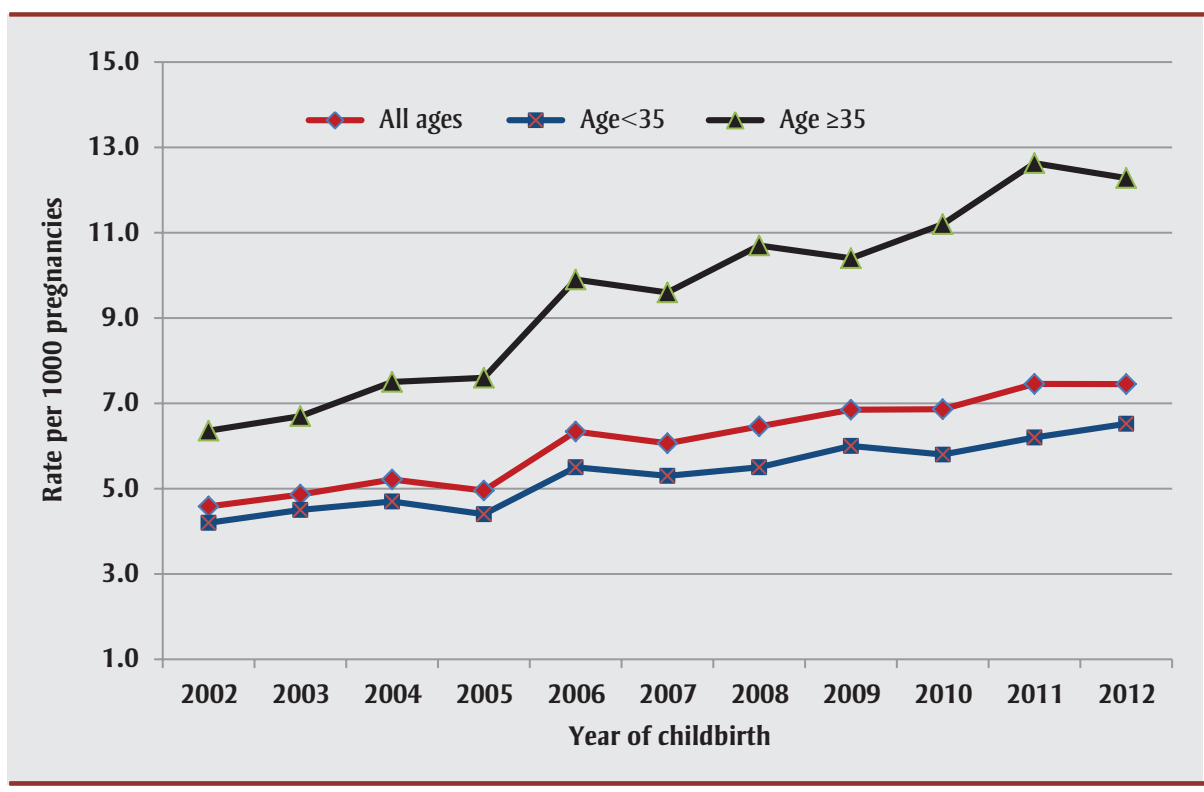

births) and type 2 DM (93.3 per 1000 live births). The adjusted OR for congenital anomalies associated with type $1 \mathrm{DM}$ in the entire population was $2.38(95 \% \mathrm{CI}$ : 2.20-2.57), while that associated with type 2 DM was 2.31 (95\% CI: 2.16-2.47). The magnitude of the association between type 1 and type 2 DM and major categories of congenital anomalies was similar except for musculoskeletal anomalies, which were not associated with type 1 DM (Table 1).

FIGURE 2

Trends in the birth prevalence of three major categories of congenital anomalies, Canada (excluding Quebec), 2002/03-2012/13

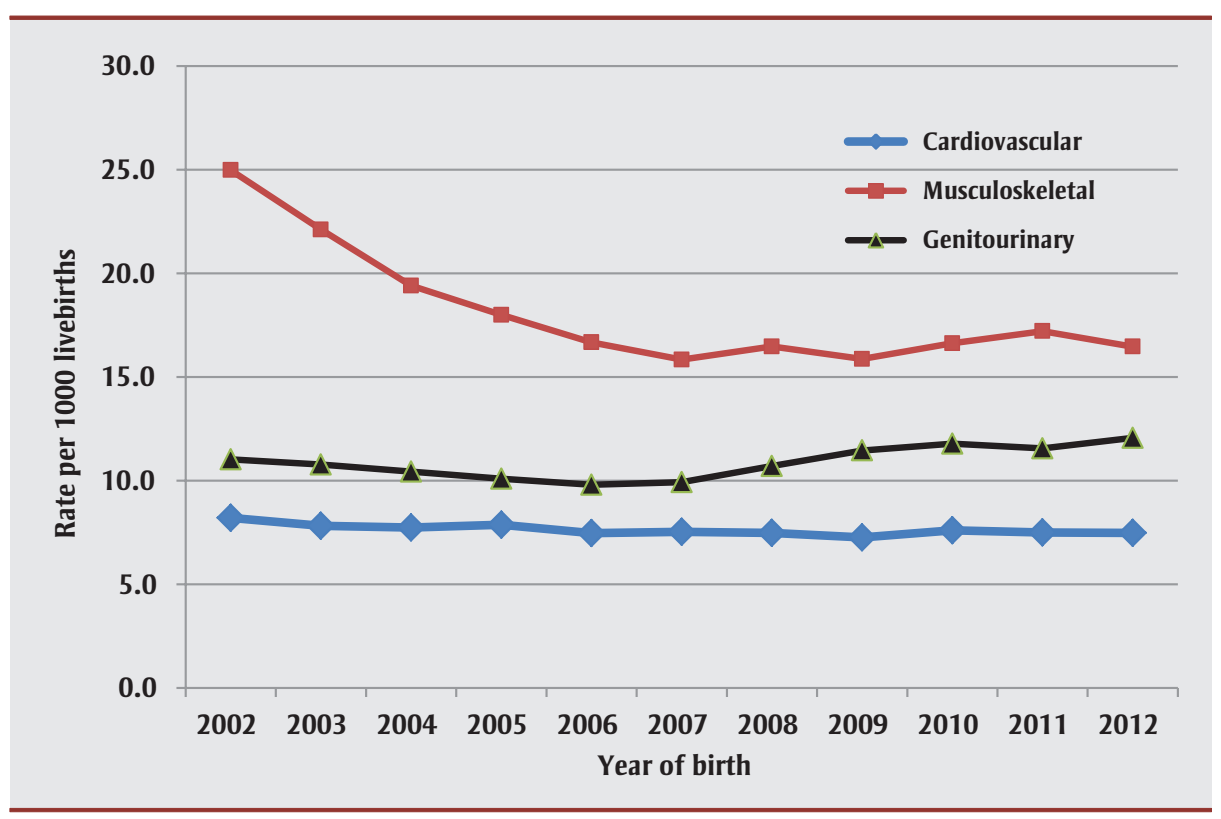

maternal pre-pregnancy DM increased from 4.6 per 1000 live births in 2002/03 to 7.5 per 1000 live births in 2012/13 while the relation between pre-pregnancy $\mathrm{DM}$ and congenital anomalies remained unchanged (the adjusted OR was 2.20 [95\% CI: $1.77-2.74$ ] in 2002/03 and 2.55 [95\% CI: 2.21-2.94] in 2012/13; $p>.05$ for the difference in adjusted ORs).

Rates of congenital cardiovascular defects were 5 times higher and rates of central nervous system anomalies were 3 times higher among women with pre-pregnancy DM compared with those without prepregnancy DM. In particular, approximately $3 \%$ of cardiovascular defects in infants could be attributed to maternal pre-pregnancy DM (Table 1). The corresponding PAR\% increased over time for cardiovascular defects, from $2.3 \%$ (95\% CI: $1.7-2.9$ ) to $4.2 \%$ (95\% CI: $3.5-4.9$ ) and for gastrointestinal defects, from $0.8 \%$ (95\% CI: $0.2-1.9)$ to $1.4 \% \quad(95 \% \quad \mathrm{CI}$ : $0.7-2.6)$, as well as for genitourinary and musculoskeletal defects (Figure 4).

\section{Discussion}

The birth prevalence of congenital anomalies in Canada was approximately 42 per 1000 live births from 2002/03 to 2012/13, and several specific congenital anomalies declined over the study period. However, exposure of fetuses to maternal prepregnancy DM increased over this 11-year period, resulting in an increase in the contribution of pre-pregnancy DM to the occurrence of congenital anomalies. Although the etiology of congenital anomalies is multifactorial and largely unknown, ${ }^{1,4,20}$ several non-inherited factors play a significant role in their development, including maternal age, pre-pregnancy DM, obesity and folate insufficiency. ${ }^{1,4,6,21-23}$ The current analysis of recent Canadian data suggests that prepregnancy DM remains a significant and modifiable risk factor.

Maternal DM has been shown to increase the risk of congenital anomalies by 2 to 5 times that of women without diabetes. ${ }^{7-11}$ Prevalence of pre-pregnancy diabetes in Ontario doubled between 1996 and 2010 among women aged 30 years and older, ${ }^{12}$ 
TABLE 1

Association between pre-pregnancy diabetes mellitus types 1 and 2 and congenital anomalies, Canada (excluding Quebec), 2002/03-2012/13

\begin{tabular}{|c|c|c|c|c|c|c|c|}
\hline \multirow[t]{3}{*}{ Anomaly type } & \multicolumn{4}{|c|}{ Prevalence (per 1000 live births) } & \multirow{2}{*}{\multicolumn{2}{|c|}{ Adjusted odds ratio $\left(95 \%\right.$ confidence interval) ${ }^{a}$}} & \multirow{3}{*}{$\begin{array}{l}\text { Population attributable } \\
\text { risk percent (PAR\%) }\end{array}$} \\
\hline & \multicolumn{2}{|c|}{ Diabetes mellitus present } & \multicolumn{2}{|c|}{ Diabetes mellitus absent } & & & \\
\hline & Type 1 & Type 2 & Type 1 & Type 2 & Type 1 & Type 2 & \\
\hline Cardiovascular & 47.4 & 41.2 & 7.5 & 7.5 & $6.55(5.89-7.29)$ & $5.35(4.83-5.89)$ & 2.97 \\
\hline Orofacial clefts & 3.6 & 3.9 & 1.4 & 1.4 & $2.48(1.70-3.63)$ & $2.77(2.02-3.80)$ & 1.03 \\
\hline Digestive & 4.7 & 4.0 & 1.6 & 1.6 & $3.06(2.20-4.25)$ & $2.41(2.76-3.29)$ & 1.06 \\
\hline Musculoskeletal & 17.6 & 26.0 & 17.8 & 17.8 & $0.99(0.84-1.18)$ & $1.49(1.32-1.69)$ & 0.18 \\
\hline Any congenital anomaly & 93.7 & 93.3 & 41.7 & 41.7 & $2.38(2.20-2.57)$ & $2.31(2.16-2.47)$ & 0.85 \\
\hline
\end{tabular}

Abbreviations: OR, odds ratio; PAR, population attributable risk.

Note: PAR\% based on the OR for diabetes mellitus (both type 1 and type 2).

${ }^{a}$ Adjusted for maternal age, number of previous births, and year of birth.

likely because of more sedentary lifestyles and higher rates of obesity. Between 2000 and 2010, the prevalence of DM in the entire Canadian population increased by $103 \%$ and is currently at $7.6 \%$; increases were especially evident among those aged 35 to 44 years. $^{23}$ Moreover, it is estimated that approximately $20 \%$ of DM cases are undiagnosed. ${ }^{24}$ The current high prevalence of both pre-pregnancy DM and overweight/ obesity $^{25}$ are of concern since both are significantly associated with an elevated risk of congenital anomalies. ${ }^{25,26}$ Specifically, fetuses of obese mothers are more likely to be affected by neural tube defects or cardiovascular anomalies compared with fetuses of mothers with a normal BMI. ${ }^{25}$

FIGURE 3

Trends in the overall birth prevalence of congenital anomalies and corresponding population attributable risk percent (PAR\%), Canada (excluding Quebec), 2002/03-2012/13

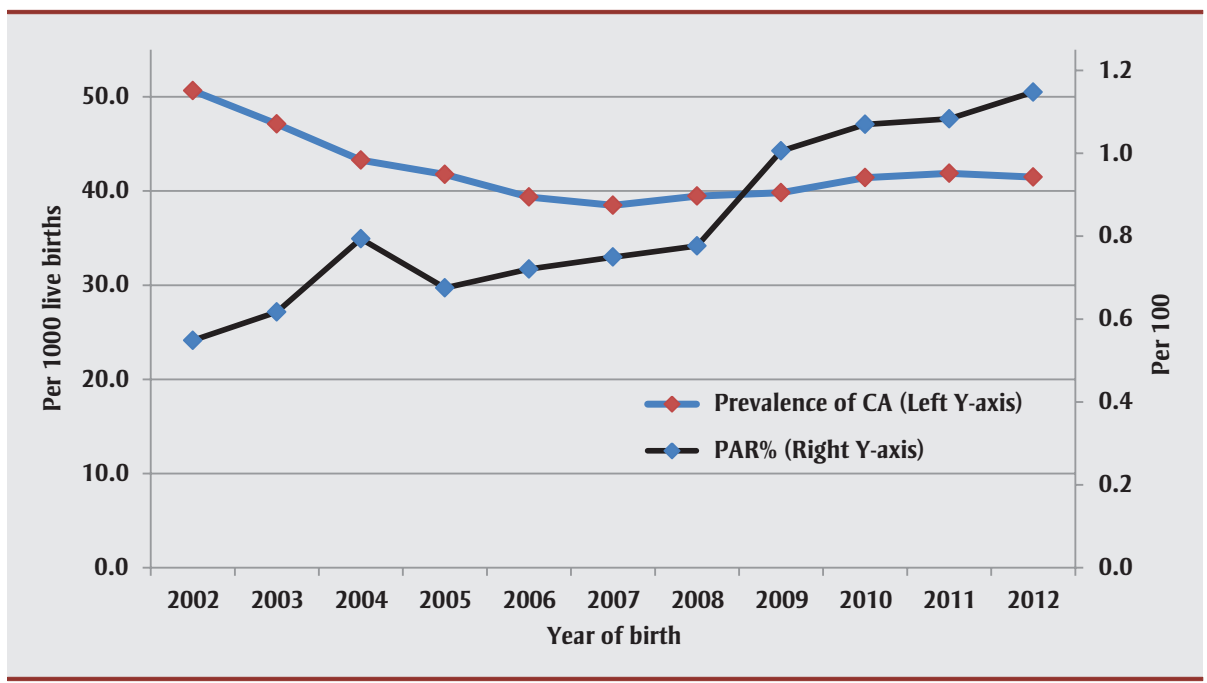

Abbreviations: CA, congenital anomaly; PAR, population attributable risk.
The association between pre-pregnancy DM and congenital anomalies we observed was consistent with other studies. ${ }^{5-11}$ For instance, Nielsen et al. ${ }^{5}$ found a prevalence odds ratio of congenital abnormalities in infants of women with diabetes of 2.1 (95\% CI: 1.5-3.1) and particularly strong associations for renal agenesis, obstructive abnormalities of the urinary tract, cardiovascular congenital abnormalities and multiple congenital abnormalities.

Periconceptional glycemic control can help mitigate the risk of congenital anomalies in women with DM. ${ }^{4,8,12,27}$ In a metaanalysis of observational studies of periconceptional glycemic control, the risk of congenital malformation increased with each standard deviation unit increase in maternal glycosylated hemoglobin concentration by an OR of 1.2 (95\% CI: 1.1-1.4). ${ }^{27}$ Studies show that preconception care is associated with a lower glycosylated hemoglobin concentration and a significantly lower risk of congenital anomalies among women with pre-pregnancy DM. ${ }^{13}$ Thus, strategies should be developed to inform women with known DM on the importance of effective preconception and prenatal health care, especially in regards to monitoring and managing blood glucose, blood pressure and target organ complications, such as retinal or renal damage. $^{13,14,28,29}$

\section{Strengths and limitations}

Our study had several limitations. First, the decline in prevalence of congenital anomalies over time is, in part, as result of increased availability of prenatal diagnosis and pregnancy termination for serious congenital anomalies. However, our data source did not have direct information about either prenatal screening or prenatal care practices, and so we were unable to exclude such effects. Since the mid-1990s, improved prenatal detection of congenital anomalies may have led to higher rates of pregnancy termination of fetuses with serious congenital anomalies, thereby leading to a decline in the proportion of live-born infants with a congenital anomaly. $2,30,31$

Second, our mother-newborn linked dataset was limited to live births; late 
FIGURE 4

Population attributable risk percent for congenital anomalies among women with pre-pregnancy diabetes mellitus, Canada (excluding Quebec), contrasting Period 1 (years 2002/03-2003/04) and Period 2 (years 2011/12-2012/13)

$$
\text { PAR \% (95\% CI) }
$$

Any anomaly, Period 10.58 (0.43 to 0.74$)$

Period 21.12 (0.94 to 1.31)

Central nervous, Period 11.67 (0.80 to 3.13$)$

Period 21.77 (0.94 to 3.00$)$

Cardiovascular, Period 12.25 (1.73 to 2.89)

Period 24.16 (3.53 to 4.89)

Cleft lip \& plate, Period 10.53 (-0.02 to 1.74)

Period 20.88 (0.18 to 2.11$)$

Gastrointestinal, Period 10.81 (0.22 to 1.90$)$

Period 21.44 (0.66 to 2.62)

Genitourinary, Period 10.43 (0.18 to 0.76$)$

Period 20.74 (0.47 to 1.07$)$

Musculoskeletal, Period 10.05 (-0.08 to 0.23$)$

Period 20.23 (0.04 to 0.45$)$

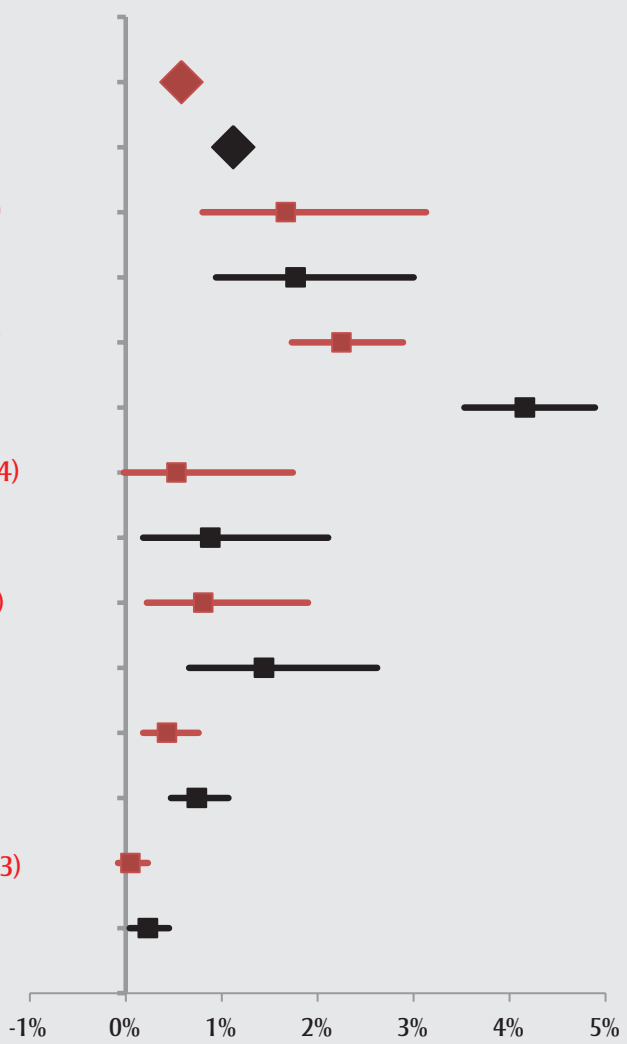

Abbreviations: $\mathrm{Cl}$, confidence interval; PAR, population attributable risk.

Note: PAR\% is calculated for all congenital anomalies and selected subcategories using the equation described in the Methods section ( $p d(O R-1) / O R$, where $p d$ is the proportion of cases exposed to the risk factor, and $O R$ is the adjusted odds ratio attributable to the risk factor. ${ }^{19}$ ), adjusted for maternal age and number of previous births obtained from separate analyses of data for the 2 indicated time periods.

pregnancy terminations and spontaneous stillbirths were not accounted for. Although how the inclusion of stillbirths would alter the association between DM and congenital anomalies is unclear, it is likely that this exclusion resulted in underestimates of the association between maternal DM (especially type $1 \mathrm{DM}$ ) and the more severe congenital anomalies caused by this condition. Further, congenital anomalies were determined within 30 days of birth, so anomalies detected in subsequent hospital readmissions were not accounted for. This may have led to underestimates of the association between maternal DM and minor congenital anomalies or those congenital anomalies that present later in infancy.
Third, no information on maternal or paternal risk factors for congenital anomalies, other than maternal age and $\mathrm{DM}$, was available in the DAD. However, lack of such information would not have impacted the temporal trends in congenital anomalies examined in this study. In terms of changes in the rate of central nervous system defects-largely comprising neural tube defects-fortification of flour with folic acid has been mandatory in Canada since 1998, and the decline in numbers of neural tube defects plateaued before the start of the study period. ${ }^{32,33}$ Further, the slight increase in congenital anomalies after 2007/08 in Canada is concordant with the increase in prepregnancy DM and maternal age
(Figures 1 and 2). Enhanced data collection among a large cohort of women with pre-pregnancy DM, with detailed ascertainment of risk factors, such as maternal obesity, would address some of the deficiencies in our study. ${ }^{1,31}$ Finally, diagnosis of congenital anomalies is typically made with knowledge of maternal DM and this may have led to an ascertainment bias, especially with regard to minor congenital anomalies.

In summary, although there has been a relative decline in the birth prevalence of congenital anomalies in Canada, a larger proportion of congenital anomalies can now be attributed to maternal pre-pregnancy DM compared with previous years. Our findings reinforce the importance of preconception care programs, particularly among women with DM, as well as of early screening in pregnancy to detect structural anomalies to guide their management. Strategies that address and prevent the occurrence of DM among women of reproductive age are also important for preventing congenital anomalies due to pre-pregnancy DM.

\section{Acknowledgements}

This study was carried out under the auspices of the Canadian Perinatal Surveillance System of the Public Health Agency of Canada. We thank the Canadian Institute for Health Information for providing access to the Discharge Abstract Database (DAD). We thank Susie Dzakpasu (Public Health Agency of Canada), Jane Evans (University of Manitoba), Michael S. Kramer (McGill University), Wei Luo, Chantal Nelson and Victoria Otterrman (all with the Public Health Agency of Canada) for comments on an earlier version of this this study. KSJ and JGR are supported by Applied Chairs awarded by the Canadian Institutes of Health Research.

\section{References}

1. Congenital anomalies in Canada 2013: a perinatal health surveillance report [Internet]. Ottawa (ON): Public Health Agency of Canada; 2013 Sep [cited 2014 Aug 6]. Available at: http://www.phac-aspc.gc.ca/ccasn-rcsac/ cac-acc-2013-eng.php. 
2. Liu S, Joseph KS, Wen SW. Trends in fetal and infant deaths due to congenital anomalies. Semin Perinatol. 2002;26:268-76.

3. Liu S, Joseph KS, Wen SW, et al. Secular trends in congenital anomaly-related fetal and infant mortality in Canada, 1985-1996. Am J Med Genet. 2001;104:7-13.

4. Jenkins KJ, Correa A, Feinstein JA, et al.; American Health Association Council on Cardiovascular Disease in the Young. Noninherited risk factors and congenital cardiovascular defects: current knowledge. A scientific statement from the American Heart Association Council on Cardiovascular Disease in the Young. Circulation. 2007;115:2995-3014.

5. Nielsen GL, Nogard B, Puho E, Rothman KJ, Sorensen HT, Czeizel AE. Risk of specific congenital abnormalities in offspring of women with diabetes. Diabet Med. 2005;22:693-6.

6. Liu S, Joseph KS, Lisonkova S, et al. Association between chronic medical conditions and congenital heart defects: a population-based cohort study. Circulation. 2013;128:583-9. DOI: 10.1161/CIRCULATIONAHA.112.001054.

7. Lisowski LA, Verheijen PM, Copel JA, et al. Congenital heart disease in pregnancies complicated by maternal diabetes mellitus: an international clinical collaboration, literature review, and meta-analysis. Herz. 2010;35:19-26.

8. Abu-Sulaiman RM, Subaih B. Congenital heart disease in infants of diabetic mothers: echocardiographic study. Pediatri Cardiol. 2004;25:137-40.

9. Wren C, Birrell G, Hawthorne G. Cardiovascular malformations in infants of diabetic mothers. Heart. 2003;89:1217-20.

10. Mikhail LN, Walker CK, Mittendorf R. Association between maternal obesity and fetal cardiac malformations in African Americans. J Natl Med Assoc. 2002;94:695-700.

11. Fuhrmann K, Reiher H, Semmler K, Fischer F, Fischer M, Glockner E. Prevention of congenital malformations in infants of insulin-dependent diabetic mothers. Diabetes Care. 1983;6:219-23.

12. Feig DS, Hwee J, Shah BR, Booth GL, Bierman AS, Lipscombe LL. Trends in incidence of diabetes in pregnancy and serious perinatal outcomes: a large, population-based study in Ontario, Canada, 1996-2010. Diabetes Care. 2014;37:1590-6.
13. Wahabi HA, Alzeidan RA, Esmaeil SA. Prepregnancy care for women with pregestational diabetes mellitus: a systematic review and meta-analysis. BMC Public Health. 2012;127:92. DOI: 10.1186/1471-2458$12-792$.

14. McLeod L, McLeod L, Ray JG. Prevention and detection of diabetic embryopathy. Community Genet. 2002;5:33-9.

15. Joseph KS, Fahey J for the Canadian Perinatal Surveillance System. Validation of perinatal data in the Discharge Abstract Database of the Canadian Institute for Health Information. Chronic Dis Can. 2009;29:96-100.

16. Liu S, Joseph KS, Hutcheon JA, et al. Gestational age-specific severe maternal morbidity associated with labor induction. Am J Obstet Gynecol. 2013;209.e1-8.

17. Kramer MS, Rouleau J, Liu S, Bartholomew S, Joseph KS for the Maternal Health Study Group of the Canadian Perinatal Surveillance System. Amniotic fluid embolism: incidence, risk factors, and impact on perinatal outcome. BJOG. 2012;119:874-9.

18. Public Health Agency of Canada. Canadian perinatal health report, 2008 edition [Internet]. Ottawa (ON): Ministry of Health; 2008 [cited 2014 Aug 6]. Available from: http:// www.phac-aspc.gc.ca/publicat/2008/cphrrspc/pdf/cphr-rspc08-eng.pdf

19. Rockhill B, Newman B, Weinberg C. Use and misuse of population attributable fractions. Am J Public Health. 1998;88:15-9.

20. Ferencz C, Loffredo CA, Correa-Villasenor A, Wilson PD, editors. Genetic and environmental risk factors of major cardiovascular malformations: the Baltimore-Washington Infant Study 1981-1989. Armonk, NY: Futura Publishing Co. 1997.

21. Czeizel AE. Periconceptional folic acid containing multivitamin supplementation. Eur J Obstet Gynecol Reprod Biol. 1998;78:151-61.

22. Watkins ML, Botto LD. Maternal prepregnancy weight and congenital heart defects in offspring. Epidemiology. 2001;12: 439-46.

23. Canadian Institute for Health Information. In due time: why maternal age matters [Internet]. 2011 [cited 2013 Sep 26]. Available from: https://secure.cihi.ca/free_products/AIB_In DueTime_WhyMaternalAgeMatters_E.pdf
24. Diabetes, by age group and sex (percent), CANSIM Table 105-0501 [Internet]. Ottawa (ON): Statistics Canada; [modified 2014 Jun12; cited 2014 Aug 6]. Available from: http://www.statcan.gc.ca/tables-tableaux/ sum-som/101/cst01/health53b-eng.htm.

25. Stothard KJ, Tennant PW, Bell R, Rankin J. Maternal overweight and obesity and the risk of congenital anomalies: a systematic review and meta-analysis. JAMA. 2009;301:636-50. DOI: 10.1001/jama.2009.113.

26. Mills JL, Troendle J, Conley MR, Carter T, Druschel CM. Maternal obesity and congenital heart defects: a population-based study. Am J Clin Nutr. 2010;91:1543-9.

27. Gueirn A, Nisenbaum R, Ray JG. Use of maternal GHb concentration to estimate the risk of congenital anomalies in the offspring of women with prepregnancy diabetes. Diabetes Care. 2007;30:1920-5.

28. Kitzmiller JL, Combs CA, Buchanan TA, Ratner RE, Kjos S. Pre-conception care of diabetes, congenital malformations, and spontaneous abortions. Diabetes Care. 1996;19:514-41.

29. Bialystok L, Poole N, Greaves L. Preconception care: call for national guidelines. Can Fam Physician. 2013;59:1037-9.

30. Liu S, Joseph KS, Kramer MS, et al. Relationship of prenatal diagnosis and pregnancy termination to overall infant mortality in Canada. JAMA. 2002;287:1561-7.

31. Joseph KS, Kinniburgh B, Hutcheon JA, et al. Determinants of increases in stillbirth rates from 2000 to 2010. CMAJ. 2013;85(8): E345-51. DOI: 10.1503/cmaj.121372.

32. Bailey LB, Berry RJ. Folic acid supplementation and the occurrence of congenital heart defects, orofacial clefts, multiple births, and miscarriage. Am J Clin Nutr. 2005;81 (suppl):1213S-7S.

33. De Wals P, Tairou F, Van Allen MI, et al. Reduction in neural-tube defects after folic acid fortification in Canada. N Engl J Med. 2007;357:135-42. 